\section{Exponential Boundedness of Nonlinear and Time-Varying Feedback Systems}

Abstract-This correspondence considers a system with a linear time-invariant part and a nonlinearity or time-varying gain in a feedback loop, and obtains conditions for the exponential boundedness of the signals, utilizing the passive operator technique. It turns out that the transfer function of the linear part shifted in argument has to satisfy the same conditions as for stability in order to ensure exponential boundedness.

\section{INTRODUCTION}

Studies in the qualitative behavior of feedback systems have thus far been mostly confined to the determination of criteria for asymptotic and input-output stability [1]. In many situations it would be of interest to have a better knowledge of the behavior of signals in the system, such as the rate of decay [2]. An attempt in this direction has been made in this correspondence by considering a system consisting of a linear time-invariant part and a nonlinear or timevarying gain in cascade in a feedback loop, and deriving conditions for the $L_{2}$ boundedness of $e_{i} \exp (\alpha t)$, where $e_{i}$ represents signals in the system.

\section{Mathematical Prelimtnaries}

Ilere detailed definitions will be omitted as these can be found in Zames [1]. $L_{2}$ boundedness will be considered throughout the correspondence.

a) $X$ denotes the input space containing functions $x$. For establishing exponential boundedness, a space $L_{2}{ }^{\alpha}$ is defined: $x \in L_{2}{ }^{\alpha}$, if $x(t) \exp (\alpha t) \in L_{L_{2}} \cdot(\alpha>0)$.

b) Linear transfer function: $G(s)$ represents the Laplace transform of the linear time-invariant operator $G$. Let $G_{a}$ and $G_{b}$ denote the operators corresponding to $G(s-\alpha)$ and $G(s+\alpha)$, respectively. It is further required that $g(t) \in L_{1}^{\alpha}$, i.e., $g(t) \exp (\alpha t)$ must be in $L_{1}$.

c) Operator $N$ : Let $N: L_{2 e} \rightarrow L_{2 e}$ be any operator having the form $N x(t)=N[x(t)]$. The operator $N$ can belong to the following classes.

1) $N \in N_{m}$ if the inequality $0 \leq(x-y)[N(x)-N(y)]<$ $K(x-y)^{2}$ holds for all $x$ and $y$ and $N(0)=0$.

2) $N \in N_{0 m}$ if $N \in N_{m}$ and $N(-x)=-N(x)$.

3) $N \in N_{t}$ if $N x(t)=f(t) x(t)$, where $0 \leq f(t)<K$ and $f(t)$ is a differentiable function.

d) Let $\mathscr{L}$ be the class of operators of the type $Z: L_{2 \varepsilon} \rightarrow L_{2 \varepsilon}$ satisfying an equation [3]

$$
Z_{x}(t)=\sum_{i=0}^{\infty} z_{i} x\left(t-\tau_{i}\right)+\int_{0}^{\infty} z(t-\tau) x(\tau) d \tau
$$

where

1) $z_{i}$ are real constants, for $i=0,1, \cdots$ and $0=\tau_{0}<\tau_{1}<, \cdots$

2) $z_{0}>0$

3) $z(\cdot)$ is a real valued function on $[0, \infty)$ and

$$
\sum_{i=0}^{\infty}\left|z_{i}\right|+\int_{0}^{\infty}|z(\tau)| d \tau<\infty
$$

\section{Statement of the Problem}

The system (Fig. 1) is described by the following set of equations:

$$
\begin{array}{ll}
e_{1}=x_{1}-y_{2}, & e_{2}=x_{2}+y_{1} \\
y_{1}=G e_{1}, & y_{2}=N e_{2}
\end{array}
$$

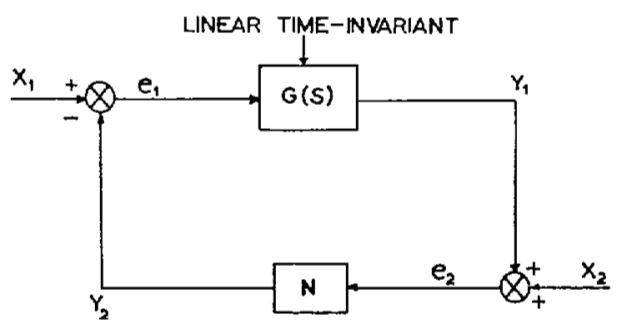

Fig. 1. Feedback system.

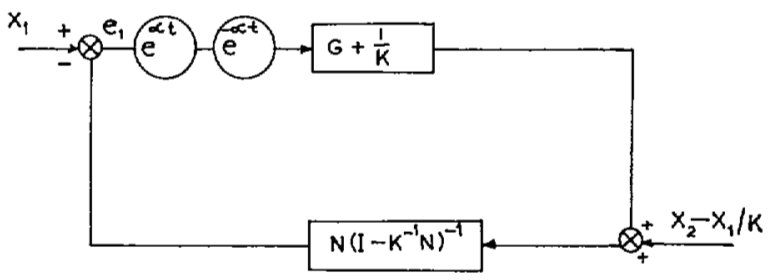

Fig. 2. Transformation.

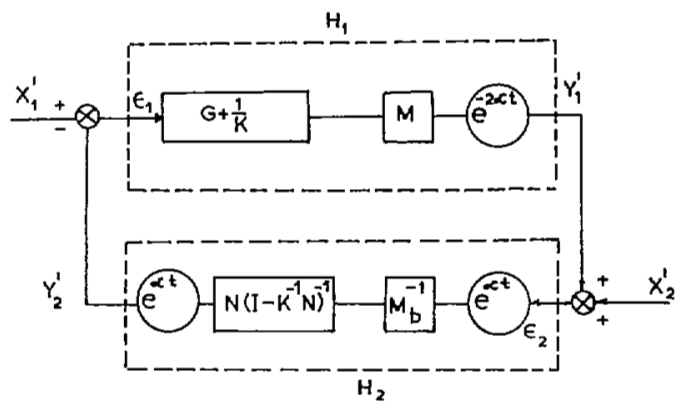

Fig. 3. Transformation.

where $G$ and $N$ are the operators defined in Section II.

Definition: The system defined by (1) is said to be exponentially bounded with order $\alpha$, if $e_{1}$ and $e_{2}$ are in $L_{2}{ }^{\alpha}$, i.e., $\left\|e_{i} \exp (\alpha t)\right\|<\infty$ and $\left\|e_{2} \exp (\alpha t)\right\|<\infty$.

The problem is to find under what conditions inputs $x_{1}, x_{2}$ belonging to $L_{2}{ }^{\alpha}$ are mapped into $e_{1}$ and $e_{2}$ which are also in $L_{2}{ }^{\alpha}$. Now, for convenience, the system is transformed in steps as shown in Figs. 2 and 3 . The transformed signals in Fig. 3 are related to the signals in the original system (1) by

$$
\begin{aligned}
\boldsymbol{\epsilon}_{1} & =\exp (\alpha t) \cdot e_{1} \\
\epsilon_{2} & =\exp (-\alpha t) \cdot M_{b}\left(e_{2}-K^{-1} y_{2}\right) \\
x_{1}{ }^{\prime} & =\exp (\alpha t) \cdot x_{1} \\
x_{2}^{\prime} & =\exp (-\alpha t) \cdot M_{b}\left(x_{2}-x_{1} / K\right) .
\end{aligned}
$$

\section{Matn Restums}

\section{Theorem 1}

Suppose there is an operator $Z$ in $\mathscr{E}$ and constants $\gamma \geq 0, \delta>0$ for which

$$
\operatorname{Re}[Z(j \omega)+\gamma j \omega][G(j \omega-\alpha)+1 / K] \geq \delta, \text { for all } \omega .
$$

If $\gamma \neq 0$, then let

$$
\lim _{|\omega| \rightarrow \infty}|\omega G(j \omega-\alpha)|=0
$$

If $N \in N_{m}$

1) $z_{i}$ (for $i \geq 1$ ) and $z(\cdot)$ are everywhere nonpositive, 


$$
\text { 2) }-\sum_{i=1}^{\infty} z_{i}-\int_{0}^{\infty} z(\tau) d \tau \leq z_{0}
$$

or if $N \in N_{0 m}$,

$$
\text { 3) } \sum_{i=1}^{\infty}\left|z_{i}\right|+\int_{0}^{\infty}|z(\tau)| d \tau \leq z_{0}
$$

then $\left\|_{i}^{\prime} e_{1} \exp (\alpha t)\right\|<\infty$ and $\left\|e_{2} \exp (\alpha t)\right\|<\infty$.

Two useful lemmas which can be easily derived are stated first.

\section{Lemma 1}

Let $P$ be any positive (strongly positive) operator; let $r$ be a time-varying gain whose derivative exists almost everywhere. Then $r P$ is positive (strongly positive) if $r$ is positive and nonincreasing almost everywhere.

\section{Lemma 2}

Let $Q$ be a positive (strongly positive) operatol; let $r$ be a timevarying gain. Then $r Q r$ is positive (strongly positive).

Proof: Let $N \in N_{m}$. Referring to Fig. 3, let $M=Z(j \omega)+\gamma \cdot j \omega$ where conditions 1) and 2) hold. Now, the inputs to the system are in $L_{2 .} H_{1}$ is strongly positive as per (3) and Lemma $1 . M_{b}(j \omega)=$ $M(j \omega+\alpha)$ and hence, $M_{b}$ also belongs to the class of allowable multipliers for $N_{m}$ [3]. Hence, $H_{2}$ is positive from Lemma 2 . Thus, from a well-known theorem of Zames [1], $\epsilon_{1}$ and $\epsilon_{2}$ are in $L_{2}$. From (2) it follows that $e_{1}$ and $e_{2}$ are in $L_{2}{ }^{\alpha}$.

The result for $N \in N_{0 m}$ can be established similarly using the corresponding multiplier. These results can be improved by extending the interval of definition of the multiplier from $[0, \infty)$ to $(-\infty, \infty)$ [4].

\section{Theorem 2}

The system (1) with $N \in N_{\text {i }}$ is exponentially bounded with order $\alpha$ if there exists an $H(s)$ such that

1) $H(s-\beta)$ is strictly positive real (SPR);

2) $H(s)[G(s-\alpha)+1 / K]$ is SPR;

3) $\dot{f} \leq 2 \beta f(1-f / K)$.

Proof: Fig. 3 reduces to a linear system $G(s-\alpha)+1 / K$ with a time-varying feedback gain $(f(t)) /(1-f(t) / K)$ when $N \in N_{t}$. The Theorem follows from earlier results [5] recast in a functional setting. Some recent results of Freedman and Zames [6] can also be used here. These involve average logarithmic variation criteria on $f(t)$ in contrast to condition 3 ).

\section{A. L. Thathachar H. S. Ranganath Dept. of Elec. Engrg. Indian Institute of Science Bangalore 12, India}

\section{REFERENCES}

[1] G. Zames "On the input-output stability of time-varying nonlinear feedback systems, pt. I: conditions derived using concepts of loop gain, conicity, and positivity" YEEE Trans. Automatic Control, vol. AC-11, pp. 228-238, April 1966.

( quine quency plane and sector nonlinearities,

[2] Control, vol. AC-1, pp. 465-476, July 1966 . 1. W. Sandberg, "On the theory of physical systems governed by nonlinear functional equations," Bell Sys. Tech. J., vol. 44, pp 871-

3] G. Zames and P. L. Falb "On the stability of systems with monotone and odd monotone nonlinearities," IEEE Trans. Automatic Control (Correspondence), pol. AC-12, pp. 221-223. April 1967.

[4] (Correspondility conditions for systems with monotone and slope restricted nonlinearities, "SIAM $J$. Control, vol. 6 , no. 1 , pp. 89108,1968 .

[5] M. Gruber and J. L. Willems, "On a generalization of the circle criterion," Proc. 4ih Ann. Allerton Conf. Circuit and System Theory. $1966, \mathrm{pp} .827-848$.

[6] M. I. Freedman and G. Zames, "Logarithmic variation criteria for the stability of systems with time varying gains," SIAM J. Control, vol. 6 , pp. $487-507$, August 1968 .

\section{Comments on "On the Optimal Angular Velocity Control of Asymmetrical Space Vehicles"}

Abstract-The purpose of this correspondence is to explain the surprisingly simple feedback laws derived by Debs and Athans in their paper, ${ }^{1}$ and to indicate a broader class of systems that can be solved by this approach.

\section{Summary of the Resuljs of Debs and Athass ${ }^{1}$}

The state equations of the system are

$$
\begin{aligned}
& \dot{x}_{1}=\alpha_{1} x_{2} x_{3}+u_{1} \\
& \dot{x}_{2}=\alpha_{2} x_{3} x_{1}+u_{2} \\
& \dot{x}_{3}=\alpha_{3} x_{1} x_{2}+u_{3}
\end{aligned}
$$

with $\alpha_{1}+\alpha_{2}+\alpha_{3}=0$.

Corresponding to a performance index which is to be minimized, given by

$J_{1}=\frac{1}{2} \int_{0}^{\infty}\left\{q\left(x_{1}^{2}+x_{2}^{2}+x_{3}^{2}\right)+(1 / q)\left(u_{1}^{2}+u_{2}^{2}+u_{3}^{2}\right)\right\} d t$

where $q>0$, the optimal feedback control law is

$$
u_{1}=-q x_{1}, \quad u_{2}=-q x_{2}, \quad u_{3}=-q x_{3} .
$$

If the performance index is

$$
\begin{aligned}
J_{2}=\int_{0}^{\infty}\left\{q \left[f_{1}\left(x_{1}\right)+\right.\right. & \left.f_{2}\left(x_{2}\right)+f_{3}\left(x_{3}\right)\right] \\
& \left.+(1 / q)\left[g_{1}\left(u_{1}\right)+g_{2}\left(u_{2}\right)+g_{3}\left(u_{3}\right)\right]\right\} d t
\end{aligned}
$$

where $f_{k}(\cdot)$ and $g_{k}(\cdot), k=1,2,3$, are positive definite functions of a single variable such that

$$
f_{k}(0)=0, \quad g_{k}(0)=0, \quad k=1,2,3, \quad q>0 .
$$

The control law which minimizes (4) is

$$
u_{1}=-q h_{1}\left(x_{1}\right), \quad u_{2}=-q h_{2}\left(x_{2}\right), \quad u_{3}=-q h_{3}\left(x_{3}\right)
$$

where $h_{k}(\cdot)$ are continuous and differentiable scalar valued functions of a single variable such that

$$
\begin{gathered}
h_{k}(0)=0, \quad h_{k}^{-1}(\cdot) \text { exists, } h_{k}\left(x_{k}\right) x_{k}>0, \quad x_{k} \neq 0, \quad k=1,2,3 \\
f_{k}\left(x_{k}\right)=\int_{0}^{x_{k}} h_{k}(x) d x
\end{gathered}
$$

and

$$
g_{k}\left(u_{k}\right)=q^{2}\left[\left(-u_{k} / q\right) h_{k}^{-1}\left(-u_{k} / q\right)-f_{k}\left(h_{k}^{-1}\left(-u_{k} / q\right)\right)\right] .
$$

\section{Explayation of the Restlis}

The results of the optimization problem considered in Section $\mathbf{I}$ become obvious if the corresponding optimization problem of an equivalent system is considered. The system equivalent to (1) for the performance indices considered is the simple decoupled linear system given by

$$
\begin{aligned}
& \dot{x}_{1}=u_{1} \\
& \dot{x}_{2}=u_{2} \\
& \dot{x}_{3}=u_{3} .
\end{aligned}
$$

Manuscript received October 9,1969 IA. S. Debs and M. A thans, IEEE Trans. Automatic Control (Short
Papers), vol. AC-14, pp. 80-83, February 1969 . 